sixteen, normal until eighteen, when it became scanty, irregular and painful, married at twenty-two, one child living, two and a half years old. Mother died of puerperal fever. Father living. There is no history of tuberculosis or tumor in the family.

She was brought to the hospital May 20, 1895; an abdominal incision was made. Adhesions were universal. A large cavity, inciuding the pelvis and left side of the abdomen, was filled with decomposing blood clot and pus. This cavity was washed as clean as possible, and filled with iodoform gauze. There were no distinct walls to the cavity. Agglutinated intestines, omentum and mesentery enclosed it for the most part. She was taken from the table in severe shock. However, she rallied and after forty-eight hours the gauze tampon was removed from the pelvis, a large glass drain introduced, and the abdominal sutures tied for the first time. An enema was ordered shortly after and it came immediately out of the glass drainage tube. For two weeks all the bowel motions were through the drainage tube. Careful attention to the fecal fistula, repeated irrigations daily, enabled us to avoid any general infection. June 2, only a small fistula remained and the patient returned home. The bowels now acted naturally.

Late in September she returned to the hospital for relief of the fistula, but after exploration and curettement it closed with little trouble. She is well and strong.

Case 7.-Mrs. Anna DeR., aged 34, married, native, was seen July 10, 1895. She had been in bed for some time suffering from abdominal distension and pain, fever, rigors and sweating. Menstruated March 24, 1895, for the last time. In the latter part of May she was suddenly attacked with severe abdominal pain, and noticed a small tumor in the left groin. This disappeared in a few hours, but the distress continued. A week later a similar attack was experienced, after which nausea was experienced and continued. Her family physician at this time discovered a hernia which was treated by a truss. The abdominal pain continned. On June 29 , an attack of vomiting, great pain and fainting occurred. She went to bed and remained there until removed to the Albany Hospital June 30, 1895. Three weeks before entering hospital flowing began and a membrane was expelled from the uterus, supposed to be a "false conception." Just prior to entering the hospital she had a chill lasting for twenty minutes. Physical examination showed the usual signs of ectopic gestation with rupture. On July 20 , she was removed to the hospital for operation the following day. During the night an attack of hemorrhage occurred and her condition became serious. The operation was made early the next morning. Blood clots and fresh blood flowed from the incision, which was at once arrested by a clamp placed on the left broad ligament. The blood, fetus and placenta were at once turned out of the abdonen. The broad ligament was immediately tied and the remnant of the left appendage removed. After the peritoneum was cleared the abdomen was filled with normal salt solution, and closed with drainage - a glass tube. The subsequent history is uneventful. The woman is now in good health.

\section{SYMPTOMS, DIAGNOSIS AND TIME FOR OPERATION IN RUPTURED TUBAL PREGNANCY.}

Read in the Section on Obstetrics and Diseases of Women, at the Fort seventh Annual Meeting of the American Medical Association, at Atlanta, Ga., May 5-8, 1896.

BY JOSEPH PRICE, M.D.

PHILADELPIIA, PA.

Careful study of the physiologic, anatomic and pathologic conditions of cases coming within our experience, while such study has not altogether removed from controversy very many subjects connected with gynecology, it has led some of us to positive convictions and to the adoption of well defined lines of practice. We are concerning ourselves less about theories, though we are not able to dispense with them altogether, but we are growing to base our rules of practice more upon the results of our observations and experiences. Pathologic systems are continually changing, one system succeeding another in quick ${ }^{\circ}$ and confusing succession. There should be no element of mere conjecture in our every day working experience. After the surgeon has discovered and relieved conditions which his experience, his observa- tion, has taught him to detect with almost mathematical certainty, the pathologist can step in and display his science in explaining cause and effect.

The occurrence of tubal pregnancy is regarded in widely different light by the theorist and the surgeon who has learned to deal with it practically, and who has accordingly come to understand the manifold directions in which speedy disaster may troop down upon unfortunate women subjected to this calamity. The argument that many cases get well of themselves, in the presence of the multitude of disasters possible, and in the light of the horror of some of these very recoveries, is so puerile that the surgeon of practical and positive bent can not regard them with complacency, nor consider that those who advance them have any authority from which to speak more positive than the vaporings of fancy. As to the causes of aberrant gestation, we are to consider them both as anatomic and moral. They may have their origin in anatomic loss of structure or in perversion of function, such as absent ciliary motion in the epithelium, or in absolute disease of the tube, or, as I have had more than once called to my attention, in the fright of illegitimate conception. Causation can rarely be determined with certainty; there are many agencies which operate to produce the trouble. The character of the attack, the whereabouts of the patient, at what employed, are always interesting considerations. The attacks are exceedingly sudden. A vigorous woman may in a few minutes look pale and exhausted and have a very feeble pulse. Any effort to change position increases the pain and she will start with a scream; the pain may be quite general and not confined wholly to the abdomen. The rational symptoms of pregnancy are not very marked. Morning sickness is never very prominent. For weeks they may complain "on and off" of sharp pain in one groin or the other. These pains are followed by bloody discharge; the odor of the discharge is also characteristic. Later the sharp and severe pain is followed by faintness and increased flow mixed with shreds and débris. Ruptures with large effusions are easily recognized upon examination. The finger detects an ill-defined boggy tumor, the uterus enlarged and posterior or pushed well to one or the other side. If the rupture is quite recent it may be difficult to determine a tumor of any character; there is simply a feeling of general resistance. In examinations made one or two days after rupture it is easy to define the irregular boggy tumor, also to locate the uterus, determine its size, position and mobility.

There is very frequently associated with these cases a history of sterility, inaptitude to conception and mild forms of pelvic disease, abortion or doubtful abortion antedating the pregnancy some four or five years, absence of one or more periods. Very frequently there is peculiar nervous disturbance, morbid apprehensions, irritability followed by acute pain, severe and recurring, pain of a variety rarely associated with other troubles. Usually the pain is followed by anemia or symptoms of concealed hemorrhage; the common symptoms of loss of blood are prominent. It is then other symptoms develop, intra-pelvic or perineal tumor due to clot or pressure, there is characteristic vesical and rectal disturbance, peculiar central fullness of the abdominal walls. Slight distension, tympany and marked tenderness rapidly follow the first rupture, recurring hemorrhage and all symptoms become more marked. The restlessness of the patient is alarming; probably 25 per cent. die in twenty 
hours, where there has not been prompt and skillful surgical relief. Hemorrhage is the real cause of death; they die both early and late in the history of the trouble; early, from rupture of tube, late or at term in primary sections done for saving both mother and a viable fetus. The non-contractile tissue of the tube favors free and continuous hemorrhage. Ruptures on the outer half of the tube or about the pavilion extremity are the least fatal. As the rupture nears the uterus the hemorrhage is most fatal. These points have been demonstrated in the experience of every one who has done any considerable number of sections. So marked has been my own observation of these facts that I commonly allude to it in my cases, exhibiting it as an object lesson to those witnessing the section, and these facts have led me to classify the cases; first, ruptures in the outer half of the tube belong to the surgeon; the second or inner half go to the coroner or coroner's physician. Rarely can you improve volume, quality and frequency of the pulse in such cases where all the symptoms are as I have narrated.

It is my conviction, fortified by my own experience, counting now one hundred and twenty-eight cases with five deaths, that the operative treatment is the only one to be considered. I am fully satisfied also that these pregnancies are rarely, if ever, in the broad ligament. In the case of fetus gone to term, in my own direct and indirect experience, the child has in no instance been in the broad ligament. I regard the chief danger of the operation as that of hemorrhage. If the patient is found so weak as to render operation an almost certain failure, I resort to salt water transfusion in order to restore the arterial tension.

Rupture with fatal hemorrhage is the most frequent termination; pyemia, septicemia and peritonitis are much rarer.

Relating to such cases Goupil says: "It is but true, I fear, that we are authorized in saying that all the cases of intra-peritoneal hemorrhage arising from extra-uterine pregnancy end in death, and although death has been delayed for six months, it is wholly exceptional. This was absolutely true in my own experience until I was emboldened-I say it-until I was shamed by Mr. Hall Wright's case into opening the abdomen and saving their lives."

The consensus of opinion by those who are competent to speak from results must be for early operation. But there are, in addition, those cases to be considered in which, after primary rupture, the fetus has still lived and advanced to full term. Here the question is one of operation with the view of saving both the life of the mother and that of the child. If one is to be lost, it is my belief that it should be that of the child; that the life of the mother is of paramount consideration. The chief danger to the mother in the operation at term in tubal pregnancy is the removal or accidental detachment of the placenta. It is easy enough to remove the child and save it, if it is viable, by operating at or near term; but the danger of fatal hemorrhage from vascular walls that can not contract, as do the uterine structures, is the vital question of the operation, so far as the mother is concerned. If we do not remove the placenta the risk of septic infection still remains.

The old and non-surgical rule of leaving the placenta to slough away is too dangerous and prolonged to be practiced. The placenta should be removed in every case, or washed and hermetically sealed, thus favoring its healthy digestion and avoiding gangrenous separation and detachment. Secondary rupture of broad ligament, discharge of placenta and fresh adhesions, or the second implantation or grafting of the placenta, have never occurred in my experience, nor have I any knowledge of such cases except that conveyed through the literature on the subject. Basing the conclusions of my judgment upon my own clinical experience, I must hold to the tubal origin and the intra-peritoneal rupture. All that follows tubal rupture is within the pelvis and peritoneal cavity, and not within the leaflets of the peritoneum forming the broad ligament.

It must be admitted that the removal of a growing and almost universally attached placenta is one of the most difficult procedures in surgery. The hemorrhage is profuse and sometimes uncontrollable; the contraction of all tissues to which it is attached simulates that of uterine tissue. Rapid separation, heat and firm pressure will commonly succeed in controlling it. As to choice of time for the operation, I am of the strong conviction that there is but one ohoice, and that is prompt removal when the accident is first recognized.

It is better to act promptly. The steps of procedure are clear and should be completed at any risk. It is better to contend primarily with the loss of blood than later with overwhelming sepsis. Tubal pregnancy is dangerous throughout its existence; the subject is never safe until surgically relieved. Exceptionally, is the trouble recognized before rupture. I have never recognized one before rupture, all before is conjecture rather than knowledge.

An important element of the history connected with these cases is that few of them are kept under observation with the definite purpose of removing the viable child at the period of spurious labor; alarming symptoms develop and subside, and consultation with a specialist, if they are at any time consulted, follows the death of the child, it rarely antedates it. Then all the conditions are found greatly aggravated by delay or neglect, or that which is infinitely worse than either or both, inexcusable ignorance. Consultations for suspected extra-uterine pregnancy are quite common in those peculiar cases of much-attenuated uterine walls in normal gestation, but the ectopic cases are permitted to pass through the primary rupture, recurring ruptures, almost constant pain and spurious labor, entailing impaired general health, without suspicion of the patient's peril.

An English authority has stated what, in connection with these cases, should be accepted as sound dictum: "As all know, the Fallopian tube is, in the vast majority of instances, the starting-point of extrauterine gestation; the most common result of this is that rupture occurs usually at the second month, through some part of the tube covered with peritoneum; a result almost universally fatal if left alone, and as invariably curable if operated on in time by abdominal section."

DISCUSSION ON PAPERS OF DRS. MACDONALD AND PRICE.

Dr. Joseph Eastman, Indianapolis - So far as I am able to judge from reading the literature of this subject and from experience, there is but one treatment for extrauterine pregnancy, and that is surgery. I will refer to one case which came under my observation because it was an extraordinary one, and it illustrates still further the futility of packing with ice and the use of electricity. A man supposed to be a competent electrician and well posted in the treatment of extrauterine 
pregnancy by electricity tried for six months to kill the fetus and failed. He then tried packing with ice for three weeks more. When the patient was in extremis there was a change of physicians. On opening the abdomen, I found the sac containing a living child in such an advanced gangrenous condition that I could not for a moment think of leaving it. Following the rule which $I$ always insist on, that we shall first find the uterus, if possible, I slipped my hand down, found the origin of the tube toward the uterus, believing that all cases of extrauterine pregnancy are primarily tubal, seized the tube at the cornu of the uterus, which felt fully as large as my wrist, and in doing so I detached a portion of the placenta from the gangrenous sac. Blood poured out freely; my assistant had gone to resuscitate the child, leaving me with a couple of nurses to do the best I could under the circumstances. Crushing down the sac (as you would crush a cherry-stone out by squeezing the cherry) with clamped forceps which 1 have with fingers like my own, I seized the sac below, then to my own astonishment I found the sac was adherent in a number of places to the intestines. With this clamp attached, after applying six ligatures around the points of hemorrhage, we got the gangrenous sac out, then quilting with iodized silk, we had the pedicle, to the cornu of the uterus, covered with iodoform collodion. Where we find a dead or living child with a gangrenous sac, the proper way to deal with such cases is to get at the cornu of the uterus either with clamps or ligature, shut off the blood supply, and remove the sac. I do not believe that it is good practice to leave the placenta to slough out. I do not believe there is a case where such men as Dr. Price would be induced to leave a gangrenous sac. I believe the sac can be removed in many cases with less risk to the patient than in leaving it or the placenta to slough out, or both combined.

Dr. C. A. L. ReED, Cincinnati-My experience has led me to attach particular importance to the symptom of shreddy metrorrhagia as an early manifestation of this condition, and I have not been able to verify the fact that this symptom occurs only after the symptoms of rupture. On the contrary, I have found that it has occurred among the earliest manifestations of pregnancy, and nodoubt it has occurred when there were no symptoms of pregnancy, and I was prompted on one occasion to make a careful study of a case and a diagnosis before rupture by following the case as suggested by the symptom, and that was one case in my experience in which a diagnosis was made before rupture, was operated upon before rupture had taken place, and the diagnosis subsequently verified by careful microscopic examination of the specimen removed. Therefore we did have in this one instance a confirmation of the fact that a shreddy metrorrhagia may occur prior to the symptoms of rupture.

It has fallen to my experience to observe so few instances, that I hesitate to allude to them, for the reason that their example has led to many errors and many fatal delays; but I have seen a number of cases in which primary rupture had occurred in the broad ligaments, for the reason that there was a definite extension of the tumor downward, and no particular extension of it upward, although its upper margins were definitely outlined. The tumor became stationary and disappeared. This was manifestly a hematocele. You may say that I had no evidence that it was a case of ectopic pregnancy, but if we are justified in saying that given cases upon which we operate were cases of ectopic pregnancy by virtue of the existence and persistence of certain sypmtoms which lead to the diagnosis, and which diagnosis is confirmed by operation, certainly we are justified in interpreting as having similar consequences a similar aggravation of symptoms, and these symptoms did exist in the few cases that I saw. While that is true, I believe it is a dangerous expedient to rely upon absorption. The maximum of safety is upon the side of operation at the earliest practicable moment, and if we have such primary rupture with such limitation of hemorrhage, the safest expedient is to avail ourselves of the quiescent interval and proceed to operate when we can do it without serious complication, and without being forced to deal with an exsanguinated patient.

Dr. James F. Baldwin, Columbus, Ohio-There was one point made by the first essayist which is of prime importance, and that is with reference to educating the general practitioner to suspect the existence of ectopic pregnancy, and thus lead to a thorough examination and to a diagnosis. It has been my fortune within the last few months to have seen seven cases of ectopic pregnancy. Two of these were seen in my own practice, the others in consultation. In five of them the diagnosis was made before rupture and operation performed. The diagnosis was subsequently confirmed, and the five patients are well to-day. Two of these cases occurred in the same patient. at an interval of six months. In each I made a presumptive diagnosis of ectopic pregnancy and operated. In two other cases no suspicion had occurred to the attending physician of ectopic pregnancy until I suggested to him that in the five cases: mentioned the diagnosis had been made by the general practitioner. This case was one of ectopic pregnancy, the diagnosis. confirmed, and the woman operated upon. In two cases the diagnosis was not made until repeated hemorrhages had occurred. The eighth case was one that was sent to the hospital during my absence, and the physician who sent the patient had made a diagnosis of peritonitis. The patient died within forty-eight. hours after admission to the hospital from peritonitis. A postmortem was made which revealed a normal uterus and tubes, but an ectopic gestation sac in the cul-de-sac of Douglas which had ruptured, producing a considerable amount of hemorrhage which resulted in peritonitis. This is an exceedingly rare condition, one which is denied by many pathologists.

We have educated the general practitioner to make a diagnosis in a large number of cases of appendicitis, and I think if we impress them in the matter of ectopic pregnancy until they suspect its existence when they have anything abnormal during the early weeks of pregnancy, they will make a diagnosis then or will have a suspicion sufficiently well grounded to send for an expert to make a thorough examination. When the general practitioner is educated up to this point cases of ectopic pregnancy will be diagnosed much earlier than they are now, and before rupture, and then the operation will be comparatively simple and few deaths will occur.

Dr. A. VAnder Veer, Albany-Papers of this kind are lessons in object teaching. They teach the general practitioner regarding the matter of an early diagnosis in these cases.

With reference to general peritonitis, inflammation of the bowel, etc., I have lived long enough to hear papers on idiopathic peritonitis, and in abdominal surgery the comparison or: difference between these terms and appendicitis have been and are presented in their true light. The general practitioner has. been taught that pelvic hematocele will be absorbed, that it. will disappear. Does it disappear? Look at the cases of pelvic: abscess-cases where a portion of bone protrudes through the vagina and rectum. Look at the cases of secondary hemorrhage and death which occur before you are fairly in the house. Some of these cases if diagnosed early and operated upon immediately would be saved. So many papers have been presented. in the past that they have mystified the general practitioner as. to the classification of this condition, as to the true pathologic: state present. What is the use of standing before the general. practitioner and arguing with him as to the form of ectopic: gestation. If you keep it up for twenty minutes your patient. is beyond relief. When the clinical symptoms are presented: the general practitioner must know that an operation is abso-. lutely necessary, and we have the authority of one man who has; perhaps operated more than any other in America in these: cases, and he tells us emphatically what ought to be done.

Stress should be laid upon the sympathetic symptoms. The: general practitioner should be educated in this matter as much, 
as he is in regard to cases of appendicitis. and in case he does not wish to operate himself he should call in a specialist to share the responsibility with him. I find that when we teach the general practitioner what to do he is not slow in following our advice.

Dr. F. J. YAGER, Camphellsburg, Ky.-I am a general practitioner, but I am firmly convinced that in these cases of ectopic pregnancy as soon as a diagnosis is made we should operate. If the general practitioner feels that he is not sufficiently expert to undertake the operation himself he should call in a specialist. The more we study these cases the more we are convinced that delay is dangerous.

Dr. Rufus B. HaLL, Cincinnati-My experience leads me to believe that a large percentage of these cases have a history of some pelvic trouble. I have known pelvic trouble to precede tubal pregnancy for at least five years in some cases. The cases are few in number in which we do not get a history of long continued pelvic trouble. We have a shorter space of time in which the patient has considered herself not entirely well since her last labor. A number of cases do not have these symptoms, but when we take the large number of cases operated on, a large per cent. of them have pelvic symptoms following some uterine or appendiceal trouble preceding their ectopic pregnancy. I have seen two women die inside of ten hours from ruptured pregnancy, before the third month of tubal gestation, as subsequently proven by autopsy. One patient lived twelve minutes after I reached the house. The time to operate is as soon as the diagnosis is made. In making a diagnosis we should not disregard the possibility of tubal pregnancy occurring in an unmarried woman, I have had two cases, one in a widow, who denied the possibility of pregnancy until after operation.

Dr. J. G. CArpenter, Stanford, Ky.--The model practitioner is a diagnostician the world over. If the general practitioner knows his business he makes a diagnosis and brings his patient to the abdominal surgeon if he does not want to operate himself. If he is the practitioner that he ought to be, he should be prepared to operate on the patient himself, because the best abdominal surgeons are made from the best general practitioners. Early diagnosis is the thing of prime importance, followed by prompt surgical interference. Operate before the patient bleeds to death from hemorrhage, before secondary lesions are set up. The patient is often unaware that she has had long standing uterine trouble. As soon as the diagnosis is made the patient should be promptly operated upon. If this was done a large number of cases that are now lost would be saved.

Dr. Mrlo B. WARD, Topeka, Kan.-We all agree that it is absolutely essential to resort to early operative interference in cases of ectopic pregnancy, particularly before rupture has taken place, if possibie. The general practitioner must be educated to the point that in cases of ectopic pregnancy it is necessary to operate as soon as a diagnosis is made. Operative interference must not be postponed. I would like to report two cases which illustrate the danger of postponing surgical interference, but I will not do so at this time.

DR. W. G. Macdonald, Albany--Regarding the general practitioner, I will say that in those cases which $I$ have seen the matter of diagnosis or suspicion of ectopic pregnancy by him has been the exception, and not the rule. We must educate the general practitioner that we have in these cases certain definite symptoms.

When we are called in consultation we sometimes give dia. metrically opposite advice in regard to what is best to be done after the diagnosis has been made, and this puts the general practitioner in trouble. We want to act together in these matters and establish uniformity of opinion.

Dr. Joseph Price, Philadelphia-The subject is not so diff. cult to understand, and a study of differential diagnosis is rather easy. From a general standpoint, the general practitioner is a very much better diagnostician than the specialist. You are in the habit of calling in specialists to do your special work; sometimes after studying your cases two or three days you come to a positive diagnosis by exclusion, and now I am going to rebuke you for permitting the specialist to take the attitude which he commonly does. After studying your case you summon a specialist, he examines your patient, shrugs his shoulders, looks wise, and says he will tell you what the condition is when he opens the abdomen. The general practitioner is as good a diagnostician in a great many cases as a specialist, and when his attention is once called to a subject he recognizes and realizes its importance. I have the greatest respect for the general practitioner, and in ninety-nine times out of one hundred I have found him usually right. If we save these patients, there is no time. for the specialist to be called in, because the operation must be done before the specialist arrives. I have said that at least 25 per cent. of the cases diewithin twenty-four hours. The symptoms are simple, physical characteristics are prominent. There is an absence of one or two menstrual periods, a delayed menstrual period, and along with this we have the characteristic agonizing pain. The attack of pain differs from any pain to which your attention has ever been called. We have the characteristic shreddy. débris which is nearly always present; a rapid pulse, and the symptoms of concealed hemorrhage. In some cases the pulse may not be bad, and the symptoms are not alarming; but if the case is an acute and typical one, cut down upon the peritoneum only, and it will be found to be black. You have only gone to. the peritoneum to make a diagnosis. You will find black blood beneath it in a large number of cases.

With reference to general practitioners making a diagnosis: in these cases, I will say that nurses occasionally after listening to discussions in the operating room and taking a record of the case, will make a correct diagnosis. I have had nurses who have sat by the bedside of patients for some time tell me that the case looks like one of extrauterine pregnancy.

\section{GAUZE AS DRAINAGE IN ABDOMINAL AND PELVIC SURGERY.}

Read in the Section on Obstetrics and Diseases of Women at the Fortyseventh Anural Meeting of the American Medical Association, held at Atlanta, Ga., May 5-8, 1896. BY MILO B. WARD, M.D. TOEHKA, KANS.

Human life so frequently depends upon the tying. of a ligature, the use of one more suture, the perfection of an operation in every minute detail, that the subject of peritoneal drainage, which is, perhaps, the most vital of all questions connected with the always grave procedure of opening the peritoneum, should never become too trite to demand careful consideration, even though much has already been written on this subject, and every phase of the question thoroughly discussed.

Viewing my failures retrospectively, I am forced to the confession that no one feature of my work has. given so much cause for regret as that of insufficient. drainage.

It is idle to say that drainage is needed only when the work has been carelessly done. However perfect. the technique may be, it is still a fact known to every operator, that there are cases whose recovery depends entirely on the drainage employed after the surgical toilet is made. The only question for consideration then is: What kind of drainage shall be used in a given case? It is so frequently necessary in choosing the material for drainage to provide means of controlling hemorrhage, thus filling two important func-. 